\title{
Metastatic malignant peripheral nerve sheath tumour. A diagnostic surprise
}

\author{
Muniza Bai ${ }^{1}$, Vishnukanth Govindaraj ${ }^{1}$, Abhishek Singh Chauhan ${ }^{1}$, Bheemanati Hanuman Srinivas ${ }^{2}$, \\ Jinkala Sree Rekha ${ }^{2}$, Vijayarangam Narenchandra ${ }^{1}$ \\ ${ }^{1}$ Department of Pulmonary Medicine; ${ }^{2}$ Department of Pathology, Jawaharlal Institute of Postgraduate Medical \\ Education and Research (JIPMER), Puducherry, India
}

\begin{abstract}
Malignant peripheral nerve sheath tumours (MPNSTs) are rare soft tissue tumours that arise from pre-existing plexiform neurofibromas or within a normal peripheral nerve. They are aggressive tumours with high rates of recurrence and distant metastases, the most common sites of metastasis being the lung followed by bone.

A 46-year-old gentleman presented with breathlessness and chest pain three years after post amputation of left thumb for an ulcerative growth. CECT thorax showed a left upper lobe mass with pleural and pericardial effusion. Within a month of presentation, he worsened and succumbed to the disease. Antemortem biopsy of the left hand ulcerative growth showed features suggestive of malignant peripheral nerve sheath tumour- epithelioid variant and post mortem liver and lung biopsy showed metastasis of MPNST. The diagnosis was a malignant peripheral nerve sheath tumour with lung, liver and cardiac metastasis.

This case report aims to highlight the importance of upfront aggressive multimodality local therapy for achieving local disease control in patients presenting with localised MPNST and regular follow up for early detection of relapse and metastasis.
\end{abstract}

Correspondence: Dr Vishnukanth Govindaraj, Associate Professor, Department of Pulmonary Medicine, Jawaharlal Institute of Postgraduate Medical Education and Research (JIPMER), Puducherry, India-605006.

Mobile number: 9894365158 . Office number: 0413-2296202.

E-mail: vishnu1429@yahoo.com

Keywords: Malignant peripheral nerve sheath tumours; neurofibromatosis; soft tissue sarcomas; cardiac metastasis; recurrence.

Acknowledgements: The authors would like to acknowledge the help rendered by Dr. Sahana and Dr. Lakshmi of the department of pulmonary medicine, JIPMER for their help in the diagnosis and workup of the patient.

Received for publication: 29 October 2020

Accepted for publication: 17 January 2021.

${ }^{\circ}$ Copyright: the Author(s), 2021

Licensee PAGEPress, Italy

Monaldi Archives for Chest Disease 2021; 91:1658

doi: 10.4081 monaldi.2021.1658

This article is distributed under the terms of the Creative Commons Attribution Noncommercial License (by-nc 4.0) which permits any noncommercial use, distribution, and reproduction in any medium, provided the original author(s) and source are credited.

\section{Introduction}

Malignant peripheral nerve sheath tumours (MPNSTs) are a rare variety of soft tissue sarcomas arising from a peripheral nerve, from a pre-existing benign nerve sheath tumour or in a patient with neurofibromatosis type 1 (NF1). It accounts for up to $5-10 \%$ of soft tissue sarcomas [1]. It is a rare and aggressive tumour with an incidence of one per million per year [2]. We herein share our experience treating a case of MPNST with local recurrence and metastasis to lung, bone, liver, and heart. This case is unique as cardiac and hepatic metastasis in MPNST has been seldom reported in the literature [3].

\section{Case Report}

A 46-year-old male, ex-smoker presented with complaints of cough with expectoration, left sided chest pain, and breathlessness for 2 weeks. He also had hoarseness of voice for the past 3 months and a painful swelling over the thenar aspect of the left hand with associated bleeding for the past 6 months (Figure 1). Upon probing further, he revealed that he had an ulcerative growth of the left thumb 3 years before and underwent amputation for the same from a different hospital. He lost follow up and presented to our institute.

In our institute, a biopsy was taken from left hand mass. Plain chest radiograph was suggestive of left upper lobe mass. CECT Thorax showed left upper lobe mass compressing left upper lobe bronchus and left main pulmonary artery, hilar and mediastinal lymphadenopathy, bilateral minimal pleural effusion, mild pericardial effusion and sclerotic vertebral metastases (Figure 2). Fibre-optic bronchoscopy showed left sided vocal cord palsy, widened carina with leftward deviation. Left upper lobe bronchus opening was not seen probably due to extrinsic compression. Lingular and lower lobe bronchi were narrowed. Transbronchial lung biopsy of left upper lobe was non-diagnostic.

He then sought alternative medicine remedy and did not turn for follow up. Subsequently he presented after a month with worsening breathlessness and weakness of both legs for 1 month duration. He denied any bladder/ bowel disturbances. On examination, he was tachypnoeic at rest. He had flaccid paraparesis (power 3/5) with absent knee and ankle reflexes. Repeat chest radiograph showed homogenous opacity in the entire left lung. CECT Thorax showed an increase in the size of left upper lobe mass extending into the mediastinum, encasing left main pulmonary artery and causing cut off of left upper lobe bronchus, hilar and mediastinal lymphadenopathy, bilateral mild pleural effusion, moderate pericardial effusion and sclerotic vertebral metastases (Figure 2). The 
liver appeared radiologically normal. NCCT Brain revealed areas of hypodensity in the caudate nucleus and internal capsule. Pericardial effusion was suggestive of cardiac metastasis. Pericardiocentesis was done. Histopathology of left-hand swelling showed features suggestive of malignant peripheral nerve sheath tumour- epithelioid variant. On immunohistochemistry, tumour cells showed strong positivity for S-100 and SOX 10 and negative for all smooth muscle markers. He was not started on palliative chemotherapy because of his poor general condition. He died within a week due to progressive disease. Morphology of post-mortem left lung and liver biopsy showed infiltration of lung tissue and liver tissue by tumour cells exhibiting an epithelioid morphology with tumour cells are positive for vimentin. The histopathology findings confirm liver and lung metastasis from left hand primary malignant peripheral nerve sheath tumour (Figure 3).

\section{Discussion}

Malignant peripheral nerve sheath tumours (MPNSTs) are defined as "any malignant tumour arising from a peripheral nerve or showing nerve sheath differentiation, excluding those originating from epineurium or peripheral nerve vasculature" [4].

It has a male adult preponderance typically in the age group of 20-50 years, though it is also reported in childhood, associated with neurofibromatosis type 1 (NF-1) [5]. The incidence of MPNSTs in the general population is $0.001 \%$; however, the incidence of malignant transformation ranges from 2 to $5 \%$ when associated with NF1 [5,6]. Approximately $50 \%$ of MPNSTs occur associated with NF-1, about $10 \%$ have a history of previous irradi- ation and the rest sporadic [7]. Plexiform neurofibromas exhibit the highest rate of malignant transformation [8]. Patients with NF-1 have an earlier presentation when compared to their sporadic coun-

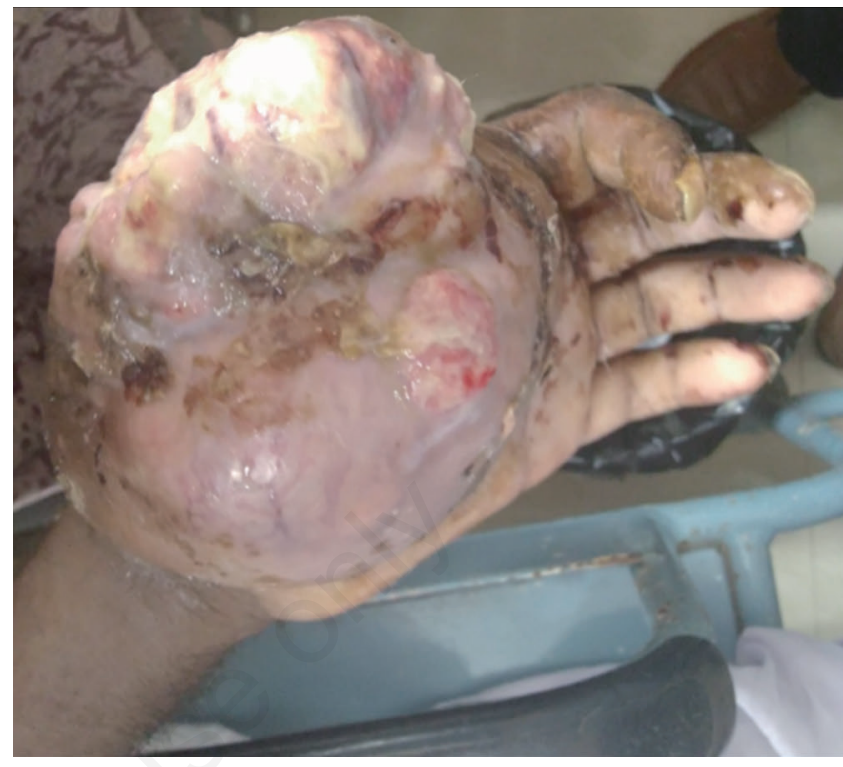

Figure 1. Single swelling of $10 \times 10 \mathrm{~cm}$ in the lateral aspect of left hand in the thenar eminence with well-defined margins, bosselated surface and multiple ulcerations over the summit of the swelling with left thumb status post amputation.
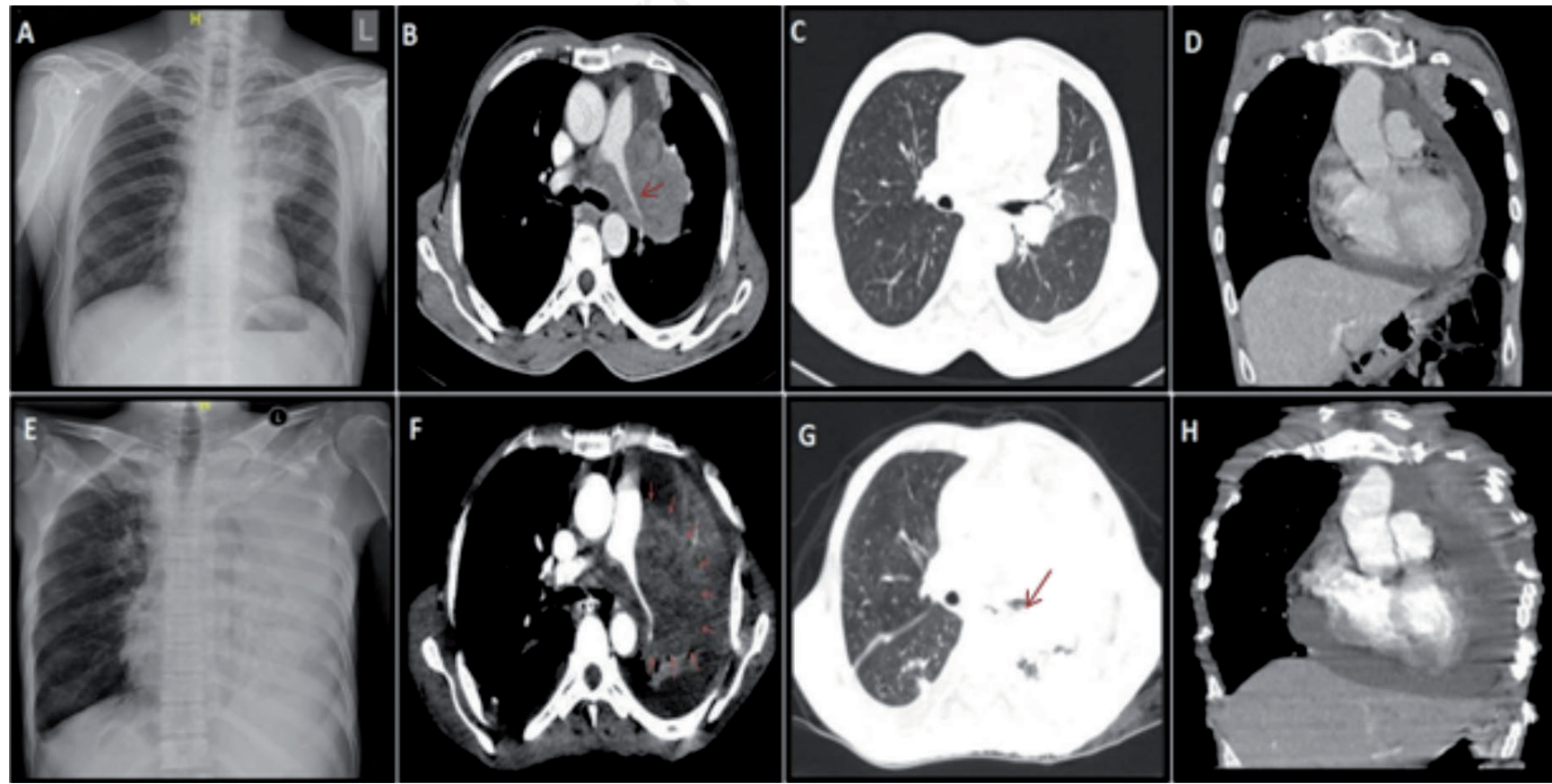

Figure 2. A-D) Images taken during first visit; E-H) images in the following visit. A) Chest X-ray: left upper lobe mass. B) CECT thorax: left upper lobe mass encasing left MPA nodal mass at the left hilar and aortopulmonary level. C) Lesion compressing the left upper lobe bronchus. D) Coronal CT showing mild pericardial effusion. E) Chest X-ray: increase in the size of left lung mass with left mild pleural effusion. F) Increase of left upper lobe mass. G) Lesion is causing cut off of left upper lobe bronchus. H) Coronal CT showing moderate pericardial effusion. 
terparts. The most common site of involvement is the extremities $(40-45 \%)$, followed by trunk (22\%), head and neck area $(21 \%)$, and retroperitoneum (15\%) [6]. The sciatic nerve is the most frequently involved.

MPNST commonly present with painless or painful enlarging mass that may be palpable or found by imaging studies. Other symptoms include paraesthesia, radicular pain or motor weakness $[5,7]$. Diagnosis is based on the constellation of clinical features, gross findings, histological, immunohistochemical, and ultrastructural features suggesting Schwann cell differentiation. MPNSTs are usually more than $5 \mathrm{~cm}$ at the time of diagnosis [5].

Microscopy typically reveals neoplastic spindle cells arranged in fascicular growth pattern, with areas of alternating hypercellularity and hypocellularity. When MPNST arises in a peripheral nerve, the tumour cells tend to track along the nerve bundles for long distances [5]. Epithelioid MPNST is a rare variant of MPNST with less than $5 \%$ of cases [9]. On immunohistochemistry, weak and patchy positivity for S-100 protein is exhibited by less than $50 \%$ cases. S- 100 protein negative status is an indicator of poor survival and high recurrence rates [10].

MRI and PET CT are important imaging modalities for assessing nerve sheath tumours. MPNSTs are intensely FDG-avid on PET CT. Whole-body MRI and PET CT help assess tumour burden of internal neurofibromas which may not be apparent on general physical examination. FDG-PET also helps distinguish high-grade MPNST from benign plexiform neurofibroma [11]. Malignant lesions have maximum standardised uptake values (SUVmax) $\geq 3.5$, whereas benign lesions have $\mathrm{SUV}<2.5$ [12]. These imaging modalities allow close surveillance of such high-risk patients enabling early diagnosis and prompt management.

MPNSTs are highly aggressive tumours with high local recurrence rate (40-65\%) and early metastasis (40-68\%), 5 and 10-year
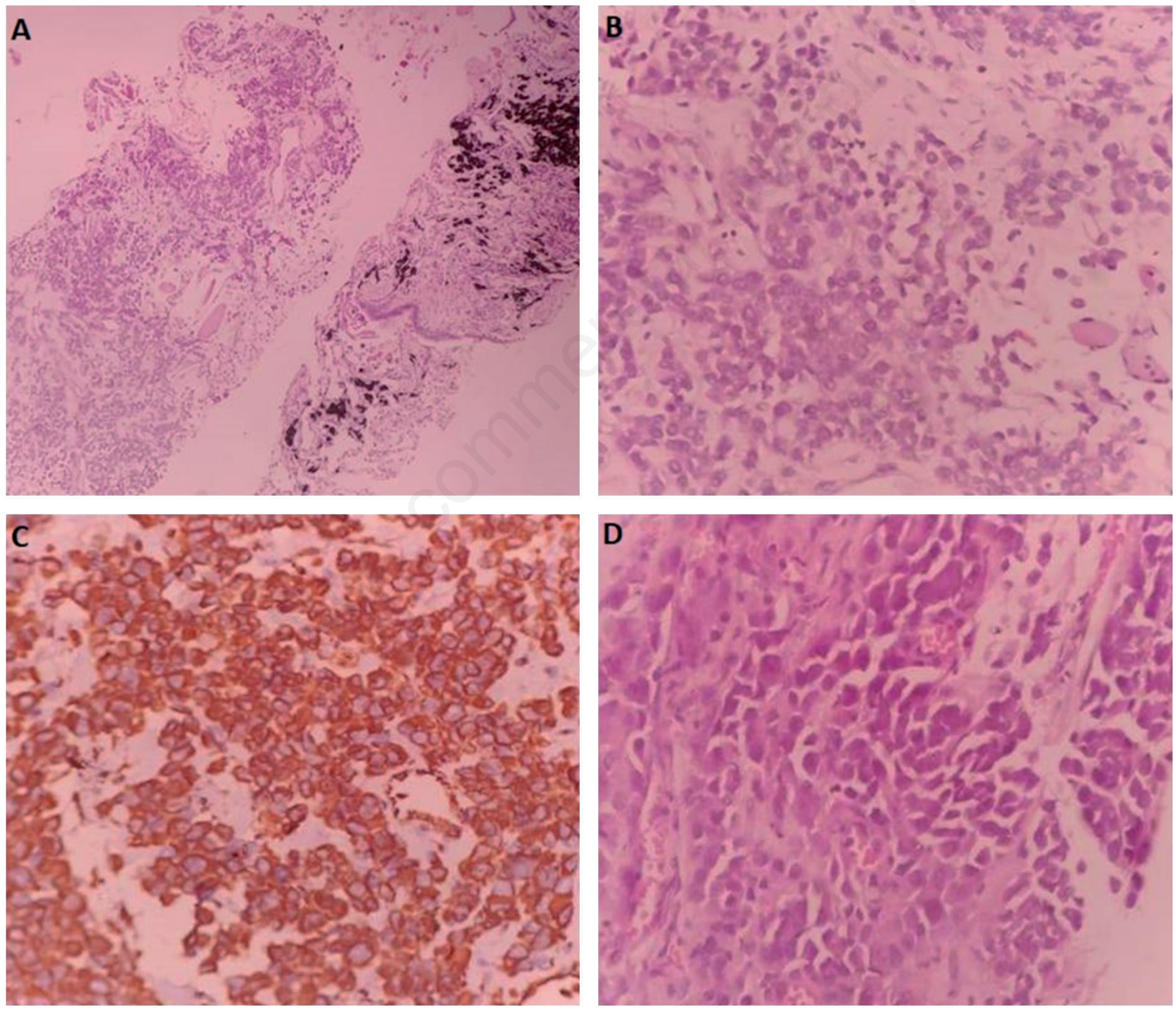

Figure 3. Photomicrographs of: A) lung tissue shows infiltration by tumour cells arranged in cords and sheets (H\&EX100); B) tumour cells are polygonal with eccentric nucleus, fine chromatin, moderate to abundant eosinophilic cytoplasm exhibiting an epitheliod morphology (H\&EX400); C) on Immunohistochemistry, tumour cells are positive for vimentin (DABX400); D) liver biopsy also showed metastasis (H\&EX400). 
survival rates being $34-60 \%$ and $22-45 \%$, respectively $[2,11]$. Truncal location is associated with poor prognosis which can be explained by the difficulty in obtaining $\mathrm{R} 0$ resection and subsequent locoregional recurrence [5]. Tumour size $>5 \mathrm{~cm}$ at presentation, unresectable tumours, high-grade lesions, nuclear P53 expression, and AKT and mTOR pathway signalling carry an indigent prognosis. However, these molecular markers have not been accepted as prognostic factors for MPNST [5,13]. Distant metastasis is usually observed in more than half the patients, the most common sites being lungs followed by bones, lymph node and liver [14].

Cardiac metastasis is a life-threatening condition and is scarcely reported [3]. Our patient had metastasis of the tumour to bone, liver, and heart. Cardiac metastasis can be classified into three types based on the site of involvement: i) pericardial or epicardial (most common site), ii) myocardial, and iii) intracavitary (least common) [5]. Clinical features are varied ranging from pericardial tamponade to constrictive pericarditis, ischemic changes on electrocardiogram, myocardial infarction, and arrhythmias. The intracavitary type is associated with features of the chamber involved. Echocardiography should be done when the presenting symptoms include chest pain, breathlessness, palpitations, or giddiness, to exclude cardiac involvement. Failure to detect cardiac involvement can result in progressive heart failure and death. Cardiac metastasis though rare should be kept in mind, as early detection of metastasis helps reduce the morbidity of the patient. These patients should be counselled in person or a telephonic follow-up is advised to detect early detection of metastasis.

Optimal management of these tumours is best achieved by a multidisciplinary approach. Aggressive surgical resection to achieve negative margins is the cornerstone of therapy and the most important prognostic factor. Radiation therapy is an important adjunct to surgery for better local control and is indicated in all intermediate to high-grade lesions and low-grade lesions after marginal excision. It may be administered as neoadjuvant or adjuvant therapy. doxorubicin-ifosfamide based chemotherapy is typically reserved for aggressive and metastatic tumours for improved systemic control, though the overall response to chemotherapy is poor, making its role questionable [1,5]. Neoadjuvant chemoradiation is usually used for originally unresectable tumours to downstage the tumour.

\section{Conclusions}

MPNST should be kept high in the differential diagnosis of rapidly progressing thoracic masses, especially in the setting of neurofibromatosis syndromes. We postulate that every case of peripheral nerve sheath tumour should be in regular follow-up for early detection of malignant transformation, local recurrence, and distant metastasis. PET CT is an integral tool that can help in close surveillance of these tumours, though further investigation to formulate an appropriate schedule is warranted.

\section{References}

1. Yoon J, Lee H, Chun J, et al. Huge intrathoracic malignant peripheral nerve sheath tumor in an adolescent with neurofibromatosis type 1. Case Rep Pediatr 2014;2014:951252.

2. Penumadu P, Shanmugasundaram S, Sachdeva A, Srinivas B. Malignant peripheral nerve sheath tumor of the thorax: Unusual location of a rare tumor. J Cancer Res Ther 2018;14:1145-8.

3. Kitamura M, Wada N, Nagata S, et al. Malignant peripheral nerve sheath tumor associated with neurofibromatosis type 1, with metastasis to the heart: a case report. Diagn Pathol 2010;5:2

4. Kleihues P, Louis D, Scheithauer B, et al. The WHO Classification of Tumors of the Nervous System. J Neuropathol Exp Neurol 2002;61:215-25. discussion 226-9.

5. Stucky C, Johnson K, Gray R, et al. Malignant peripheral nerve sheath tumors (MPNST): The Mayo Clinic Experience. Ann Surg Oncol 2011;19:878-5.

6. Grobmyer S, Reith J, Shahlaee A, et al. Malignant peripheral nerve sheath tumor: Molecular pathogenesis and current management considerations. J Surg Oncol 2008;97:340-9.

7. Ducatman B, Scheithauer B, Piepgras D, et al. Malignant peripheral nerve sheath tumors. A clinicopathologic study of 120 cases. Cancer 1986;57:2006-21.

8. Wise J, Cryer J, Belasco J, et al. Management of head and neck plexiform neurofibromas in pediatric patients with neurofibromatosis type 1. Arch Otolaryngol Head Neck Surg 2005; 131:712-8.

9. Jo V, Fletcher C. Epithelioid malignant peripheral nerve sheath tumor. Am. J. Surg. Pathol. 2015;39:673-2.

10. Wang T, Yin H, Han S, et al. Malignant peripheral nerve sheath tumor (MPNST) in the spine: a retrospective analysis of clinical and molecular prognostic factors. J Neurooncol 2015;122:349-5.

11. Kamran S, Shinagare A, Holler Howard S, et al. Intrathoracic malignant peripheral nerve sheath tumors: imaging features and implications for management. Radiol Oncol 2013;47: 230-8.

12. Goldblum JR, Weiss SW, Folpe AL. Enzinger and Weiss's Soft Tissue Tumors. 6th ed. Philadelphia: Elsevier Saunders; 2014. p. 855-79.

13. Zou C, Smith K, Liu J, et al. Clinical, pathological, and molecular variables predictive of malignant peripheral nerve sheath tumor outcome. Ann Surg 2009;249:1014-22.

14. Treglia G, Taralli S, Bertagna F, et al. Usefulness of wholebody fluorine-18-fluorodeoxyglucose positron emission tomography in patients with neurofibromatosis type 1: A systematic review. Radiol Res Pract 2012;2012:431029. 